\title{
Factores de riesgo de trastornos conductuales y emocionales en la niñez: estudio comunitario en el Uruguay
}

\author{
Robert Kohn, ${ }^{1}$ Itzhak Levav, ${ }^{2}$ Paulo Alterwain, ${ }^{3}$ Gloria Ruocco, ${ }^{3}$ \\ Myriam Contera ${ }^{4}$ y Sheri Della Grotta ${ }^{1}$
}

RESUMEN Objetivos. Explorar algunos de los factores de riesgo demográficos que se asocian con la presencia de problemas conductuales o emocionales en los niños, y examinar la posibilidad de una relación entre el estrés psicológico en los padres y trastornos psicológicos en los hijos.

Materiales y métodos. En el presente estudio la presencia de estrés psicológico en los padres y ciertas características psicosociales que aumentan el riesgo de sufrir trastornos mentales en la niñez fueron examinados en una muestra de niños uruguayos de dos comunidades urbanas y una rural. La investigación, que se llevó a cabo en Ciudad Vieja y Barrio Sur, en Montevideo, y en el pueblo de Colonia de Sacramento, abarcó a 115 niños entre las edades de 5 y 15 años. Las madres contestaron por sus hijos el Cuestionario de Morbilidad Psiquiátrica Infantil (QMPI), instrumento para la detección de problemas conductuales que podrían ser indicio de trastornos emocionales en los niños. Adicionalmente, ambos padres proporcionaron la información demográfica solicitada en la Psychiatric Epidemiology Research Interview Demoralization Scale (PERI-D) [Escala de Desmoralización para la Investigación Epidemioló-gica en Psiquiatría]; contestaron el cuestionario CAGE ${ }^{5}$ para el tamizaje del alcoholismo; se sometieron al Social Support Network Inventory [Inventario de la Red de Apoyo Social], y respondieron preguntas sobre su propio estado de salud mental.

Resultados. Cincuenta y tres por ciento de los niños tuvieron puntajes mayores de 6 en el QMPI, resultado que señala la presencia de problemas conductuales o emocionales. La autopercepción de un trastorno emocional y de desmoralización en las madres mostró una asociación significativa con un mayor riesgo de problemas conductuales o emocionales en los hijos.

Discusión. A juzgar por nuestros resultados, la salud mental del niño es un problema social y sanitario cuya epidemiología debe explorarse más a fondo en el Uruguay.

Palabras clave Salud mental, Uruguay, infancia, problemas conductuales.

Después de la restauración de la democracia en el Uruguay en 1987, el Ministerio de Salud, con el apoyo de la Organización Panamericana de la Salud (OPS), lanzó un programa nacional para la protección de la salud

3 Ministerio de Salud Pública, Dirección General de la Salud, Unidad de Salud Mental, Montevideo, Uruguay.

Departamento de Psiquiatría y Conducta Humana de Brown University, Hospital Butler, Providence, Rhode Island, EE.UU. Dirección postal: Brown University, Department of Psychiatry and Human Behavior, Butler Hospital, 345 Blackstone Blvd., Providence, RI 02906, EE.UU. Tel.: (401) 455-6277; fax: (401) 455-6566; correo electrónico: Robert_Kohn @brown.edu

2 Ministerio de Salud, Tel Aviv, Israel
4 Universidad de la República, Departamento de Medicina Preventiva y Social, Facultad de Medicina, Montevideo, Uruguay.

5 La sigla viene de attempts to cut down on drinking, annoyance with criticisms about drinking, guilt about drinking, and using alcohol as an eye-opener. mental. Este programa tenía un componente epidemiológico destinado a determinar la frecuencia y distribución de los trastornos mentales en adultos y niños.

Numerosos factores propios del ambiente familiar pueden aumentar el riesgo que corre un niño de tener problemas conductuales o emocionales. La disfunción familiar $(1,2)$-la presencia de conflictos entre los padres, la 
disrupción de la interacción entre padres e hijos y la presencia de factores que interfieren con la crianza de los hijos - pone en peligro el desarrollo social y emocional del niño en general. Está demostrado que el conflicto entre los padres, junto con los sucesos vitales adversos, coloca a los niños en mayor riesgo de sufrir problemas emocionales (3). Factores que menoscaban el desarrollo de una sana interacción entre padres e hijos han sido documentados en progenitores con problemas de alcoholismo y son los que más influyen en la conducta de los hijos, especialmente de los varones pequeños $(1,4)$.

En otros estudios también se ha investigado si un trastorno psiquiátrico en el padre o la madre interfiere con las prácticas de crianza (5-10). En general, estos estudios han revelado que los niños criados por padres depresivos tienen tasas más altas de problemas de conducta. Además de las circunstancias que rodean al niño en su entorno familiar, otros factores adversos en la comunidad también pueden afectarlo. La pobreza (11), el hacinamiento (12) y el alojamiento en una vivienda subsidiada (13) son algunos de ellos.

En América Latina se han hecho pocas encuestas para evaluar la salud mental de los niños. En Salvador, Bahia, Brasil, se llevó a cabo un estudio comunitario en el cual se aplicó el Questionario de Morbilidade Psiquiatrica Infantil (QMPI) (14). Según los resultados, $23,2 \%$ de los niños entre las edades de 5 y 14 años tenían problemas emocionales.

En Puerto Rico se han llevado a cabo varios estudios epidemiológicos sobre la salud mental infantil. El primero consistió en una encuesta a domicilio realizada por muestreo en dos etapas (15), durante la cual se administró la Child Behavior Checklist (CBCL) [Lista de Conductas Infantiles] a madres y maestros, seguida del Diagnostic Interview Schedule for Children (DISC) [Instrumento de Entrevista Diagnóstica para Niños] $(16,17)$ a quienes tuvieron resultados positivos. Este estudio, que abarcó a niños de 4 a 16 años de edad, informó una prevalencia de trastornos mentales de $45,3 \%$ en un período de 6 meses con la CBCL, 40,5\% al aplicar los criterios del DSM-III sin discapacidad y 17,9\% al aplicar los criterios del DSM-III más discapacidad. ${ }^{6}$

La segunda investigación formó parte del Methods for the Epidemiology of Child and Adolescent Mental Disorders [MECA] Study [Estudio del Instituto Nacional de Salud Mental de los Estados Unidos sobre los Métodos Epidemiológicos Aplicados a los Trastornos Mentales de los Niños y los Adolescentes], que se llevó a cabo en Puerto Rico (19) y otras partes de los Estados Unidos. Se administró el DISC a niños de 9 a 17 años en la comunidad, con lo cual se detectó una prevalencia de trastornos mentales de $12,3 \%$ en un período de 6 meses cuando se aplicaron criterios de discapacidad, y de 39,4\% cuando tales criterios no fueron aplicados. En México, el Reporting Questionnaire for Children (RQC) [Cuestionario de Notificación Infantil] (20), instrumento para el tamizaje de problemas conductuales en los niños, se aplicó como parte de la Encuesta Nacional de Salud Mental (21). Sobre la base de la información aportada por los padres, se estimó una prevalencia de 15,6\% en niños de 3 a 12 años de edad. En Chile, varias investigaciones sobre la salud mental de los niños se han realizado con instrumentos diagnósticos semiestructurados que se han basado en la Décima Clasificación Internacional de Enfermedades (CIE-10) (22, 23). En niños de 5 a 7 años, la tasa de trastornos fue de $43 \%$. En un estudio de una muestra representativa de la población de escolares de primaria en Santiago, Chile, la tasa de prevalencia aproximada de problemas conductuales y emocionales fue de $15 \%$ (24).

Los objetivos del presente estudio fueron los siguientes: 1) explorar algunos de los factores de riesgo demográficos que se asocian con la presencia de

\footnotetext{
6 La determinación de discapacidad se basa en la Children's Global Assessment Scale [Escala de Evaluación General para Niños] (18). Un puntaje mayor de 60 en esta escala indica la presencia de discapacidad como consecuencia del trastorno mental.
}

problemas conductuales o emocionales en los niños; y 2) examinar la posibilidad de una relación entre el estrés psicológico en los padres y trastornos psicológicos en los hijos.

\section{MATERIALES Y MÉTODOS}

\section{Sujetos}

Las personas que participaron en esta encuesta provinieron de dos localidades: Montevideo, cuya población excede el millón de habitantes, y Colonia de Sacramento, con 19100 habitantes según el Censo Nacional de 1987. Se hizo un muestreo en dos distritos de Montevideo, Ciudad Vieja y Barrio Sur, cuya población combinada es de más de 80000 habitantes. En ambos distritos de Montevideo se seleccionó cada quinto domicilio en siete corredores censales. Participaron 213 domicilios, con un total de 551 hombres, mujeres y niños mayores de 5 años. En Colonia de Sacramento, los sujetos provinieron de cada cuarta casa en 11 corredores censales, con lo cual se obtuvo un total de 534 sujetos en 174 domicilios.

Inicialmente se entrevistó a todos los adultos en los domicilios seleccionados y la madre proporcionó información sobre cada uno de sus hijos. No obstante, para el análisis se eligió aleatoriamente a un solo niño en cada domicilio, eliminándose así la posibilidad de un sesgo de selección. En total la muestra se compuso de 115 niños menores de 15 años. Los padres de cada niño elegido aleatoriamente fueron identificados a fin de estudiar como posibles factores de riesgo sus rasgos sociodemográficos y su estado emocional.

\section{Instrumentos}

En este estudio se aplicó el Questionario de Morbidade Psiquiatrica Infantil, que fue creado para investigar la morbilidad psiquiátrica en una zona urbana de Salvador, Bahía, Brasil, y para detectar trastornos mentales infantiles 
(25). ${ }^{7}$ El QMPI fue completado por 115 madres con hijos de 5 a 14 años con el fin de detectar en estos niños problemas conductuales o emocionales que apuntaran a la presencia de un trastorno psiquiátrico. El instrumento consistió en 35 preguntas sobre la presencia de problemas del desarrollo, problemas conductuales, trastornos neuróticos y psicosomáticos, enfermedades orgánicas del cerebro y retraso mental. Las respuestas a estas preguntas eran dicotómicas (sí o no). Al usarse un punto de corte de 6 , los estudios brasileños de validación del QMPI mostraron una sensibilidad de $88 \%$, una especificidad de $83 \%$ y una tasa de errores de clasificación de 15\%. Por consiguiente, las repuestas al QMPI fueron valoradas en términos de puntaje total y usando el 6 como punto de corte.

Los padres proporcionaron información sobre su estado civil, escolaridad, ingresos familiares, religión, ocupación, discapacidades físicas y problemas emocionales autopercibidos. Cada uno de los padres completó la PERI-D (26) y el CAGE (27) y contestó preguntas sobre las fuentes de apoyo social durante el año anterior, según el Social Support Network Inventory [Inventario de la Red de Apoyo Social] (28).

Se usó la PERI-D como indicador aproximado de la presencia de trastornos mentales en los padres. La PERI-D se ha definido como un instrumento que sirve para hacer un tamizaje de la población en general y obtener submuestras de personas de distinto sexo, clase social y grupo étnico, que padecen síntomas de gravedad (26). La PERI-D tiene la limitación, sin embargo, de que aunque mide la intensidad de muchas de las manifestaciones de enfermedad mental, no puede arrojar un diagnóstico preciso (29). En un estudio piloto que se llevó a cabo en el Uruguay en personas con y sin enfer-

\footnotetext{
El QMPI es el único instrumento aplicado en este estudio que fue creado para el medio brasileño; todos los demás fueron creados en Estados Unidos. La versión española de la PERI-D fue validada en el Uruguay; el CAGE y el Social Network Inventory han sido aplicados en poblaciones hispanas.
}

medades mentales, a la PERI-D se le encontró una sensibilidad de $76 \%$ y una especificidad de $72 \%$ con un punto de corte de 1,3. Los resultados de ese estudio, que recogió datos sobre la población adulta del Uruguay, indican que $23 \%$ de la misma estaba en riesgo de sufrir un trastorno mental (30).

El segundo intrumento administrado a los adultos fue el cuestionario CAGE con cuatro componentes, el cual se usa para identificar a personas con problemas de alcoholismo en la población. Los componentes del CAGE fueron incluidos en un grupo de preguntas sin contenido comprometedor - ¿Pierde el apetito con frecuencia? ¿Cuál es su bebida predilecta? - a fin de reducir las probabilidades de deshonestidad en las respuestas. El CAGE también detecta casos de sospecha de alcoholismo, pero no permite hacer el diagnóstico específico. Dos o más respuestas afirmativas indican que la sospecha es fuerte; una sola respuesta afirmativa es motivo para que un médico haga una investigación más minuciosa (27). Los datos arrojados por este estudio, que se llevó a cabo con una muestra de 387 adultos, indican que 10,9\% de la población tuvo por lo menos una respuesta afirmativa en el CAGE.

La autodenuncia de problemas emocionales entre los adultos fue valorada mediante una respuesta afirmativa a cualquiera de las dos preguntas siguientes: ¿Ha sido usted tratado alguna vez o ha acudido a un profesional por problemas psicológicos, como el nerviosismo? A quienes respondieron que no, se les hizo esta pregunta adicional: ¿Cree usted que en algún momento ha debido recibir tratamiento por problemas psicológicos? Una de las dos preguntas fue contestada afirmativamente por $25,5 \%$ de la muestra. La encuesta fue realizada en 1989 por entrevistadores contratados por el Ministerio de Salud del Uruguay.

\section{Análisis de los datos}

Con el fin de explorar los factores de riesgo de trastornos psicológicos en los niños, se tuvieron en cuenta las siguientes variables: edad y sexo del niño, número de personas en el hogar, ciudad de residencia, y variables paternas y maternas, tales como estado civil, escolaridad, prácticas religiosas, apoyo social percibido, prestigio ocupacional e ingresos. La asociación entre estos factores y el puntaje en el QMPI fue examinada mediante modelos de regresión mútiple y logística. Para determinar si existía una relación entre la salud mental de los padres y el estado psicológico del hijo, la relación entre el puntaje de los padres en la PERI-D, sus puntajes en el CAGE y su propia percepción de sus problemas emocionales, por un lado, y los problemas conductuales de los hijos por el otro, fue examinada usando el QMPI como variable independiente en modelos de regresión logística.

Se aplicaron métodos de regresión por eliminación de variables en sentido retrógrado con el fin de elegir los mejores modelos explicativos, usando como posibles factores de riesgo los detallados en el párrafo anterior. El criterio para incluir una variable en el modelo fue la obtención de un valor $P<0,05$ y, para dejarla en el modelo, la obtención de un valor $P<0,1$. El paquete estadístico SUDAAN (31) se usó con pesos, haciendo ajustes por la probabilidad de un sesgo en las contestaciones del encuestado.

\section{RESULTADOS}

La muestra consistió en un total de 115 niños, de los cuales 53 (46,1\%) eran de sexo femenino y 60 de sexo masculino (no se anotó el sexo de dos de los niños). Las edades oscilaron entre los 5 y los 14 años, con una media de 9,3 \pm 2,7. El número de miembros de familia varió de 2 a 11 y tuvo un promedio de $4,3 \pm 1,6$. El cuadro 1 presenta algunas características adicionales de la muestra. No se encontraron diferencias estadísticamente significativas en los datos demográficos, la edad, la escolaridad, la religión y los ingresos de los padres.

Los puntajes en el QMPI variaron de 0 a 23,0, siendo la media 6,8 $\pm 4,0$; el 
CUADRO 1. Características demográficas de la muestra $(n=115)$. Montevideo y Colonia Sacramento, Uruguay

\begin{tabular}{|c|c|c|}
\hline Característica & No. & $\%$ \\
\hline \multicolumn{3}{|l|}{ Sexo del hijo } \\
\hline Masculino & 60 & 52,2 \\
\hline Femenino & 53 & 46,2 \\
\hline \multicolumn{3}{|l|}{ Ciudad } \\
\hline Montevideo: Ciudad Vieja & 23 & 20,0 \\
\hline Montevideo: Barrio Sur & 30 & 26,1 \\
\hline Colonia de Sacramento & 62 & 53,9 \\
\hline \multicolumn{3}{|l|}{ Edad (media $\pm D^{a}$, en años) } \\
\hline Hijo & $10,1 \pm 2,7$ & $\ldots$ \\
\hline Madre & $39,0 \pm 8,9$ & $\ldots$ \\
\hline Padre & $43,4 \pm 9,4$ & $\ldots$ \\
\hline Miembros del hogar (media $\pm \mathrm{DE}$ ) & $4,3 \pm 1,6$ & $\ldots$ \\
\hline \multicolumn{3}{|l|}{ Escolaridad materna ${ }^{b}$} \\
\hline Primaria & 30 & 26,1 \\
\hline Secundaria & 29 & 25,2 \\
\hline Universidad & 36 & 31,3 \\
\hline Ninguna & 7 & 6,1 \\
\hline Desconocida & 13 & 11,3 \\
\hline \multicolumn{3}{|l|}{ Escolaridad paterna $^{\mathrm{c}}$} \\
\hline Primaria & 29 & 31,5 \\
\hline Secundaria & 21 & 22,8 \\
\hline Universidad & 27 & 29,3 \\
\hline Ninguna & 4 & 4,3 \\
\hline Desconocida & 11 & 12,0 \\
\hline \multicolumn{3}{|l|}{ Afiliación religiosa materna } \\
\hline Sí, pero no practicante & 41 & 35,7 \\
\hline Sí, practicante estricta & 17 & 14,8 \\
\hline Sí, practicante parcial & 36 & 31,3 \\
\hline Ninguna & 18 & 15,9 \\
\hline Desconocida & 3 & 2,6 \\
\hline \multicolumn{3}{|l|}{ Afiliación religiosa paterna } \\
\hline Sí, pero no practicante & 34 & 37,0 \\
\hline Sí, practicante estricto & 4 & 4,3 \\
\hline Sí, practicante parcial & 21 & 22,8 \\
\hline Ninguna & 26 & 28,3 \\
\hline Desconocida & 7 & 7,6 \\
\hline \multicolumn{3}{|l|}{ Estado civil materno } \\
\hline Casada & 88 & 76,5 \\
\hline Otro & 25 & 21,7 \\
\hline Desconocido & 2 & 1,7 \\
\hline \multicolumn{3}{|l|}{ Esrado civil paterno } \\
\hline Casado & 87 & 94,6 \\
\hline Otro & 4 & 4,3 \\
\hline Desconocido & 1 & 1,1 \\
\hline \multicolumn{3}{|l|}{ Ingresos maternos } \\
\hline Bajos & 5 & 4,3 \\
\hline Moderados-bajos & 21 & 18,3 \\
\hline Moderados-altos & 64 & 55,7 \\
\hline Altos & 20 & 17,4 \\
\hline Desconocidos & 5 & 4,3 \\
\hline \multicolumn{3}{|l|}{ Ingresos paternos } \\
\hline Bajos & 3 & 3,3 \\
\hline Moderados-bajos & 17 & 18,5 \\
\hline Moderados-altos & 51 & 55,4 \\
\hline Altos & 17 & 18,5 \\
\hline Se desconocen & 4 & 4,3 \\
\hline
\end{tabular}

\footnotetext{
a $\mathrm{DE}$ = Desviación estándar

${ }^{\mathrm{b}}$ Número de madres $=115$.

c Número de padres $=92$.
}

puntaje medio de los varones fue de $7,2 \pm 4,3$ y el de las niñas, $6,2 \pm 3,6$. Cuando se aplicó un punto de corte de 6 en el QMPI, 53,0\% (61) de la muestra sacó un puntaje mayor de 6 , y la diferencia entre los varones $(52 \%, 31)$ y las niñas $(40 \%, 21)$ no fue estadísticamente significativa. Ser residente de Colonia de Sacramento mostró una asociación significativa con la obtención de puntajes mayores de 6 en el QMPI $\left(63 \%, \chi^{2}=14,44 ;\right.$ grados de libertad [gl] $=2 ; P<0,0008)$.

$\mathrm{Al}$ tratarse como valores continuos, los puntajes de los niños y las niñas en el QMPI no mostraron diferencias significativas. No se hallaron asociaciones significativas entre los factores de riesgo demográficos de los padres y los puntajes en el QMPI. La ciudad de residencia fue el único factor de riesgo que mostró una asociación significativa con puntajes más altos en el QMPI $(F=6,73 ; \mathrm{gl}=2 ; P<0,002)$. La prueba de Tukey reveló que los niños que vivían en cualquiera de las dos localidades de Montevideo tenían un puntaje medio significativamente menor que el de los niños que vivían en Colonia de Sacramento. En los modelos de regresión logística, la ciudad de residencia fue la única variable demográfica que mostró una asociación estadísticamente significativa con el puntaje en el QMPI.

El puntaje de la madre en la PERI-D mostró una correlación con el puntaje en el QMPI como valor continuo $(r=$ $0,29$; $P<0,002)$, mientras que el de los padres no mostró dicha correlación $(r=0,10 ; P=\mathrm{ns})$. Además, los puntajes de padres y madres en la PERI-D no mostraron ninguna correlación entre sí $(r=0,17 ; P=\mathrm{ns})$. El puntaje de las madres en la PERI-D, como variable continua y categórica, se asoció con puntajes más altos en el QMPI. En los modelos de regresión logística con eliminación retrógrada de variables, el puntaje en la PERI-D y el lugar de residencia fueron los únicos factores de riesgo asociados significativamente con puntajes maternos más altos en el QMPI (cuadros 2 y 3).

En el caso de los padres, pero no de las madres, cuando el puntaje en el 
CUADRO 2. Riesgo relativo de problemas conductuales o emocionales en el hijo, según puntaje materno en la PERI-D y lugar de residencia. Montevideo y Colonia de Sacramento, Uruguay

\begin{tabular}{lccc}
\hline & $\beta$ & EE $^{\mathrm{a}}$ & $P$ \\
\hline Puntaje materno en la PERI-D & 2,26 & 0,78 & 0,005 \\
$\quad$ Colonia de Sacramento & $\ldots$ & $\ldots$ & $\ldots$ \\
Montevideo & & & 0,009 \\
$\quad$ Ciudad Vieja & $-2,60$ & 0,98 & 0,003 \\
$\quad$ Barrio Sur & $-2,49$ & 0,82 & \\
\hline
\end{tabular}

Nota: Valores estadísticos del modelo: $R^{2}=19,8 \% ; F=87,90,3 / 111 ; P<0,001$.

${ }^{a} \mathrm{EE}=$ error estándar

${ }^{b}$ Grupo de referencia.
QMPI se examinó como variable continua, se encontró una asociación significativa con la autopercepción de problemas emocionales $(\beta=2,31$; error estándar $=1,10 ; P<0,04)$. Cuando se incorporó en los modelos de regresión logística la autopercepción de problemas emocionales, la ciudad de residencia fue el único otro factor de riesgo significativo (cuadro 4). No se detectó ningua asociación estadísticamente significativa entre el puntaje en el QMPI como variable continua y el puntaje de madres o padres en el CAGE.

\section{DISCUSIÓN}

Mediante esta pequeña encuesta comunitaria se encontró que alrededor de $53 \%$ de la muestra de niños tenía un puntaje mayor de 6 en el QMPI, lo cual apunta a la posible presencia de problemas conductuales o emocionales. Aunque este porcentaje es mayor que el hallado en otros estudios a base de instrumentos aplicados a niños, concuerda, por ejemplo, con la encontrada en estudios en que se ha aplicado la CBCL $(37,8 \%)$ (15). Las tasas en este estudio son más altas que las que se hallaron en el estudio efectuado en el Brasil con el QMPI (14); la diferencia se debe, probablemente, al hecho de que en nuestro estudio se aplicó una escala dicotómica en lugar de la escala de Likert utilizada en el estudio brasileño.

Las tasas obtenidas con el QMPI deben ser interpretadas con cautela, ya que son el resultado de un solo tamizaje; es necesario efectuar un segundo procedimiento diagnóstico para confirmar la presencia de un trastorno mental.
CUADRO 3. Riesgo relativo de problemas conductuales o emocionales en el hijo, según puntaje materno $>1,3$ en la PERI-D y lugar de residencia. Montevideo y Colonia de Sacramento, Uruguay

\begin{tabular}{lccc}
\hline & $\beta$ & EE $^{\mathrm{a}}$ & $P$ \\
\hline Puntaje materno en la PERI-D $>1,3$ & $\ldots$ & & \\
$\quad$ Nob & $\ldots, 17$ & $\ldots$ & $\ldots$ \\
Sí & $\ldots$ & 1,07 & 0,004 \\
$\quad$ Colonia de Sacramento & & $\ldots$ & $\ldots$ \\
Montevideo & $-2,48$ & 0,94 & 0,01 \\
$\quad$ Ciudad Vieja & $-2,92$ & 0,85 & 0,001 \\
$\quad$ Barrio Sur & &
\end{tabular}

Nota: Valores estadísticos del modelo: $R^{2}=20,1 \% ; F=87,35,3 / 111 ; P<0,001$.

a $\mathrm{EE}=$ error estándar.

${ }^{b}$ Grupo de referencia.
En estos niños, el único factor de riesgo sociodemográfico asociado con puntajes más altos en el QMPI fue el ser residente de la Colonia de Sacramento, y no de Montevideo. No se sabe con exactitud a qué se debe la diferencia entre estas comunidades. La presencia de un sesgo al seleccionar la muestra no se puede descartar, pese a la falta de diferencias significativas entre un lugar y otro en cuanto a los rasgos demográficos de padres e hijos.

Los problemas emocionales en los padres mostraron una relación directa significativa con la presencia de dificultades conductuales o emocionales en el hijo. La salud mental de los adultos también mostró diferencias en función del sexo. Entre los padres, la autopercepción de tener problemas emocionales $\mathrm{y}$, entre las madres, la de sentirse desmoralizadas, se asociaron con mayores problemas conductuales o emocionales en el hijo. Es factible que, de haber sido mayor el tamaño muestral, la autopercepción de problemas emocionales en la madre también hubiera mostrado una asociación estadísticamente significativa; ese análisis tuvo un poder de apenas $48 \%$, en comparación con $56 \%$ en el caso del de los padres.

Los resultados aquí descritos no difieren mucho de los que han arrojado otros estudios latinoamericanos sobre la salud mental infantil. En un estudio realizado en una población urbana de Salvador, Bahía, Brasil, en el cual se aplicó el QMPI (32), el puntaje materno en esta prueba (33), que es un índice del estado psicológico, mostró una correlación con la presencia de problemas conductuales en el hijo. En cambio, no se encontró tal correlación con el puntaje paterno en el QMPI. Ese estudio, a diferencia del nuestro, también mostró una relación directa entre el tamaño de la familia y la edad del niño por un lado, y puntajes más altos en la QMPI por el otro.

En el estudio efectuado en Puerto Rico $(34,35)$, los hijos de padres con trastornos emocionales sacaron puntajes más altos en el CBCL. Esta asociación perduró aun después de hacer ajustes por los factores de riesgo pro- 
CUADRO 4. Riesgo relativo de problemas conductuales o emocionales en el hijo, según la autopercepción paterna de tener problemas de salud mental y según lugar de residencia. Montevideo y Colonia de Sacramento, Uruguay

\begin{tabular}{lccc}
\hline & $\beta$ & $\mathrm{EE}^{\mathrm{a}}$ & $P$ \\
\hline Autopercepción paterna de tener problemas de salud mental & & \\
$\quad \mathrm{No}^{\mathrm{b}}$ & $\ldots$ & $\ldots$ & $\ldots$ \\
Sí & 2,03 & 1,05 & 0,05 \\
Colonia de Sacramento & $\ldots$ & $\ldots$ & $\ldots$ \\
$\quad$ Montevideo & & & 0,21 \\
$\quad$ Ciudad Vieja & $-1,46$ & 1,15 & 0,01 \\
$\quad$ Barrio Sur & $-2,56$ & 1,0 & \\
\hline
\end{tabular}

Nota: Valores estadísticos del modelo: $R^{2}=12,3 \% ; F=72,28,3 / 111 ; P<0,001$.

${ }^{a} \mathrm{EE}=$ error estándar.

${ }^{b}$ Grupo de referencia.

pios del ambiente familiar. A diferencia de lo encontrado en nuestro estudio, no solo la depresión, sino también el alcoholismo, mostraron una asociación con la desadaptación del hijo, independientemente del sexo del progenitor.

En el estudio mexicano, que fue similar al nuestro, los síntomas de depresión basados en el CES-D (36) mostraron una asociación con mayores problemas conductuales solamente en el caso de las madres (37). En cambio, Caraveo et al. (38) hallaron una asociación directa entre un consumo excesivo de alcohol en los padres y la presencia de trastornos conductuales en los hijos. En el estudio uruguayo, la falta de una asociación entre problemas de alcoholismo en los progenitores y problemas conductuales en los hijos podría deberse a que el CAGE es un instrumento de tamizaje y no un instrumento diagnóstico.

Debido a las notables discrepancias entre los informes proporcionados por padres e hijos en cuanto a la naturaleza e intensidad del trastorno psicológico, todo un corpus bibliográfico duda de la validez de la salud mental materna como factor pronóstico de la salud mental de los hijos $(39,40)$. Esto despierta interrogantes sobre la idoneidad metodológica de estudios basados exclusivamente en las declaraciones de las madres sobre su propia salud. Najman et al. (41) encontraron que la presencia de un trastorno mental en la madre en el momento de la encuesta tenía un notable efecto en su notificación de problemas conductuales en los hijos, lo cual arroja dudas sobre la validez de los informes proporcionados por personas deprimidas. Fergusson y Lynskey (42), sin embargo, llegaron a la conclusión de que cuando se tenían en cuenta como factores de confusión la disfunción conyugal y la deprivación social, las asociaciones entre la depresión materna y los problemas conductuales en los hijos se reducían a un mínimo carente de significación estadística. Postularon que ello reflejaba la presencia de circunstancias familiares y sociales que generaban síntomas depresivos en las mujeres, los cuales, a su vez, de por sí aumentan los riesgos de que los hijos adopten conductas rebeldes. No obs- tante, otros informes han planteado la posibilidad de que las madres deprimidas perciban con más agudeza los problemas de comportamiento de sus hijos (43).

La enfermedad mental del padre o de la madre puede constituir un factor de riesgo genético y ambiental para el hijo (44), cuya relación con los demás puede afectar a su autoestima, a sus vínculos afectivos y a sus facultades cognoscitivas, factores que a la larga podrían vincularse con un cuadro sintomático (45).

La salud mental infantil, en sus muchos aspectos, es asunto de interés para la salud pública. A fin de superar las limitaciones del presente estudio, la próxima generación de estudios de investigación en epidemiología psiquiátrica en América Latina deberán basarse en muestras más grandes y en el uso de instrumentos estandarizados aplicados a niños, y no solo a los padres y maestros, para poder establecer cifras de prevalencia. Asimismo, en el futuro las investigaciones analíticas deberán incorporar en su diseño factores de riesgo genéticos y ambientales, así como factores que ejercen un posible efecto protector sobre el bienestar emocional de los niños. Estos últimos suelen omitirse de la planificación de investigaciones y programas porque existe la noción equivocada de que los trastornos que sufren los niños son leves y transitorios y que su efecto es poco profundo. Si se han de comprender mejor las necesidades de los niños, también es necesario llevar a cabo estudios longitudinales.

Agradecimiento. Los autores agradecen a Vladimiro Rathauser, antiguo representante de la OPS en Uruguay, y a todos los entrevistadores el apoyo que han brindado a esta investigación.

\section{REFERENCIAS}

1. Quinton D, Rutter M, Liddle C. Institutional rearing, parenting difficulties and marital support. Psychol Med 1984;14(1):107-124.

2. Rae-Grant N, Thomas BH, Offord DR, Boyle $\mathrm{MH}$. Risk, protective factors, and prevalence of behavioral and emotional disorders in children and adolescents. J Am Acad Child Adolesc Psychiatry 1989;28(2):262-268.

3. Hetherington EM, Cox M, Cox R. Long-terms of divorce and remarriage on the adjustment of children. J Am Acad Child Adolesc Psychiatry 1985; 24(5):518-530.

4. Avison WR. Risk factors for children's conduct problems and delinquency; the significance of family milieu. Trabajo presentado en 
la American Society of Criminology Annual Meeting, Nueva Orleans, 1992.

5. Caplan HL, Cogill SR, Alexandra H, Robson KM, Katz R, Kumar. Maternal depression and the emotional development of the child. Br J Psychiatry 1989;154:818-822.

6. Downey G, Coyne JC. Children of depressed parents: an integrative review. Psychol Bull 1990;108(1):50-76.

7. Hammen C, Gordon D, Burge D, Adrian C, Jaenicke C, Hiroto D. Maternal affective disorders, illness and stress: risk for children's psychopathology. Am J Psychiatry 1987;144(6): 736-741.

8. Merikangas KR, Prusoff BA, Weissman MM. Parental concordance for affective disorders: psychopathology in offspring. J Affect Disord 1988;15(3):279-290.

9. Rutter M, Quinton D. Parental psychiatric disorder: effects on children. Psychol Med 1984; 14(4):853-880.

10. Weissman MM, Warner V, Wickramaratne $\mathrm{P}$, Moreau D, Olfson M. Offspring of depressed parents, 10 years later. Arch Gen Psychiatry 1997;54(10):932-940.

11. Offord DR, Boyle MH, Fleming JE, Blum HM, Grant NI. Ontario child health study. Summary of selected results. Can J Psychiatry 1989; 34(6):483-491.

12. Rutter M, Quinton D. Psychiatric disorder: ecological factors and concepts of causation. En: McCurck H, ed. Ecological Factors in Human Development. Amsterdam: North Holland Publishing; 1977.

13. Rutter M. Stress, coping and development: some issues and some questions. J Child Psychol Psychiatry 1981;22(4):323-356.

14. Almeida-Filho N. Epidemiologia das Desordens Mentais da Infancia no Brasil. Salvador: Centro Editorial e Didatico da UFBA; 1985.

15. Bird HR, Canino G, Rubio-Stipec M, Gould MS, Ribera J, Sesman M, et al. Estimates of the prevalence of childhood maladjustment in a community survey in Puerto Rico. Arch Gen Psychiatry 1988:45(12):1120-1126.

16. Achenbach T, Edelbrock C. Behavioral problems and competencies reported by parents of normal and disturbed children ages four through sixteen. Monogr Soc Res Child Dev 1981;46(1):1-82.

17. Costello AJ, Edelbrock C, Dulcan MK, Kalas $\mathrm{R}$, Klaric SH. Development and testing of the NIMH Diagnostic Interview Schedule for children in a clinic population. Final Report (contract RFP-DB-81-0027). Rockville, MD: Center for Epidemiologic Studies, NIMH; 1984.

18. Shaffer D, Gould MS, Brasic J, Ambrosini P, Fisher P, Bird H, et al. A Children's Global Assessment Scale (CGAS). Arch Gen Psychiatry 1983;40(11):1228-1231.

19. Shaffer D, Fisher P, Dulcan MK, Davies M, Piacentini J, Schwab-Stone ME, et al. The NIMH Diagnostic Interview Schedule for Children version 2.3 (DISC-2.3): description, acceptability, prevalence rates and perfor- mance in the MECA study. J Am Acad Child Adolesc Psychiatry 1996;35(7):865-877.

20. Giel R, de Arango MV, Climent CE, Harding $\mathrm{TW}$, Ibrahim $\mathrm{HH}$, Ladrido-Ignacio $\mathrm{L}$, et al. Childhood mental disorders in primary health care: results of observations in four developing countries. A report from the WHO Collaborative Study on Strategies for Extending Mental Health Care. Pediatrics 1981; 68(5): 677-683.

21. Caraveo J, Medina-Mora ME, Tapia R, Rascon L, Gómez M, Villatoro J. Trastornos psiquiátricos en niños de la República Mexicana. Resultados de una encuesta de hogares. Anales del Instituto Mexicano de Psiquiatría 1992;3:56-62.

22. Minoletti A, López C. Las enfermedades mentales en Chile: magnitud y consecuencias. Santiago, Chile: Departamento Programa de las Personas, Unidad de Salud Mental: República de Chile, Ministerio de Salud, División de Salud de las Personas; 1999.

23. Toledo V, de La Barra F, López C, George M, Rodríquez J. Diagnósticos psiquiátricos en una cohorte de escolares de primer año de enseñanza básica del área occidente de Santiago. Revista Chilena Neuropsiquiatria 1997;35: 17-24

24. Bralio S, Seguel X, Montenegro H. Prevalencia de trastornos psíquicos en la población escolar de Santiago de Chile. Acta Psiquiatr Psicol Am Lat 1987;33(4):316-325.

25. Almeida-Filho N. Development and assessment of the QMPI: a Brazilian children's behaviour questionnaire for completion by parents. Soc Psychiatry 1981;16:205-211.

26. Dohrenwend BP, Shrout PE, Egri G, Mendelsohn FS. Nonspecific psychological distress and other dimensions of psychopathology. Arch Gen Psychiatry 1980;37(11):1229-1236.

27. Ewing J. Detecting alcoholism. The CAGE Questionnaire. JAMA 1984;252(14):1905-1907.

28. Flaherty JA, Gaviria FM, Pathak DS. The measurement of social support: the Social Support Network Inventory. Compr Psychiatry 1983; 24(6):521-529.

29. Roberts RE, Vernon SW. Usefulness of the PERI-Demoralization Scale to screen for psychiatric disorder in a community sample. Psychiatry Res 1981;5(2):183-193.

30. Kohn R, Alterwain P, Levav I, Ruocco G, Murillo N, Contrera M, Carzoli L, et al. Screening for mental illness and alcohol use disorders in two communities in Uruguay. (En preparación).

31. Shah BV, Barwell BG, Bieler GS. SUDAAN. Software for the statistical analysis of correlated data. Release 7.5. Research Triangle Park, NC: Research Triangle Institute; 1997.

32. Almeida-Filho N, Santana V, de Souza AL, Jacobina RR. Relaçoes entre a saude mental dos pais e a saúde mental das crianças em uma população urbana de Salvador-Bahia. Acta Psiquiatr Psicol Am Lat 1985;31(3):211-221.

33. Santana VS. Estudo epidemiologico das doenças mentais em um bairro de Salvador - Nor- deste de Amaralina. Salvador: Tese de Mestrado em Saude Comunitaria-UFBA; 1978.

34. Canino G, Bird H, Rubio-Stipec M, Bravo M, Alegria M. Children of parents with psychiatric disorder in the community. J Am Acad Child Adolesc Psychiatry 1990;29(3):398-406.

35. Rubio-Stipec M, Bird H, Canino G, Bravo M, Alegria M. Children of alcoholic parents in the community. J Stud Alcohol 1991;52(1): 78-88.

36. Radloff LS. The CES-D Scale: A self report depression scale for research in the general population. Applied Psychological Measurement 1977;1:385-401.

37. Caraveo J, Medina-Mora ME, Villatoro J, Rascon ML. La depresión en el adulto como factor de riesgo en la salud mental de los niños. Salud Mental 1994:17:56-60.

38. Caraveo J, Medina-Mora ME, Villatoro J, Rascon ML, Martínez-Vélez NA. El consumo de alcohol en adultos como factor de riesgo asociado con trastornos psíquicos en los niños. Salud Mental 1995;18:18-24.

39. Weissman MM, Wickramaratne $P$, Warner $V$, John K, Prusoff BA, Merikangas KR, et al. Assessing psychiatric disorders in children. Discrepancies between mothers' and children's reports. Arch Gen Psychiatry 1987;44(8): 747-753.

40. Webster-Stratton C, Hammond M. Maternal depression and its relationship to life stress, perceptions of child behavior problems, parenting behaviors, and child conduct problems. J Abnorm Child Psychol 1988;16(3): 299-315.

41. Najman JM, Williams GM, Nikles J, Spence S, Bor W, O'Callaghan M, et al. Mothers' mental illness and child behavior problems: causeeffect association or observation bias? J Am Acad Child Adolesc Psychiatry 2000;39(5): 592-602.

42. Fergusson DM, Lynskey MT. The effects of maternal depression on child conduct disorder and attention deficit behaviours. Soc Psychiatry Psychiatr Epidemiol 1993;28(3): 116-123.

43. Richters J, Pellegrini D. Depressed mothers' judgments about their children: an examination of the depression-distortion hypothesis. Child Dev 1989; 60(5):1068-1075.

44. Schwartz S, Dohrenwend BP, Levav I. Non-genetic familial transmission of psychiatric disorders? Evidence from the children of the Holocaust. J Health Soc Behav 1994;35(4): 385-402.

45. Minde K. Effect of disordered parenting on the development of children. En: Lewis M, ed. Adolescent psychiatry. Second edition. Baltimore: Williams and Wilkins; 1996.

Manuscrito recibido el 21 de noviembre de 2000. Aceptado para publicación el 14 de marzo de 2001. 
ABSTRACT Objectives. To explore some of the demographic risk factors that are associated with the risk of behavioral or emotional problems in children, and to examine the possibility of an association between psychological stress in the parents and psychological problems in their children.

Risk factors for behavioral and emotional problems in childhood: a community study in Uruguay

Methods. In this study, the presence of psychological stress in the parents and certain psychosocial characteristics that increase the risk of mental problems in childhood were examined in a sample of Uruguayan children from three communities, two urban and one rural. The study, which was carried out in Ciudad Vieja and Barrio Sur, in the city of Montevideo, and in Colonia de Sacramento, a rural town, comprised 115 children between the ages of 5 and 15. Mothers answered the Child Psychiatric Morbidity Questionnaire (QMPI), an instrument for the detection of behaviors pointing to the presence of emotional problems in children. Both parents also supplied the demographic information requested in the Psychiatric Epidemiology Research Interview Demoralization Scale; they answered the CAGE questionnaire, a screening tool for alcoholism; they responded to the Social Support Network Inventory; and they answered questions about their self-perceived mental health.

Results. Fifty-three percent of the children had scores greater than 6 on the QMPI, which indicates the possible presence of behavioral or emotional problems. Fathers' self-perception of emotional problems and mothers' feeling of being demoralized showed a statistically significant association with a greater risk of behavioral or emotional problems in their children.

Conclusions. In light of our results, mental health in childhood is a social and public health problem that warrants further epidemiologic study in Uruguay.

\section{BECAS DE INVESTIGACIÓN DEL INSTITUTO GORGAS}

Fecha límite: 1 de agosto de 2001

日 Instituto Gorgas de Medicina Tropical y Preventiva patrocina una serie de becas que serán otorgadas por la American Society of Tropical Medicine and Hygiene para financiar investigaciones colaborativas entre instituciones investigadoras de México, Centroamérica, el Caribe, el norte de Sudamérica (Bolivia, Colombia, Ecuador, Perú, Guyana, Suriname, Guyana Francesa y Brasil) y Estados Unidos de América.

Los candidatos deben ser licenciados en medicina o doctorados en algún campo relacionado con las enfermedades tropicales, poseer la ciudadanía de alguno de los países mencionados y tener un puesto en alguna institución médica, educativa, de investigación o de salud pública en esos países. En principio, también deben tener menos de 40 años. Las becas no sue len superar los US\$25000.

Las candidaturas deben presentarse antes del 1 de agosto de 2001 y deben incluir el proyecto de investigación, el curriculum vitae, dos cartas oficiales en apoyo del proyecto de investigación, una de la institución en la que trabaje el candidato y otra de la institución que lo vaya a recibir y la documentación que demuestre que el proyecto ha sido aprobado por los correspondientes comités institucionales. De cada uno de estos cinco documentos se enviarán cinco copias.

Las candidaturas y preguntas deben dirigirse a: Gorgas Memorial Institute Research Award

American Society of Tropical Medicine and Hygiene

60 Revere Drive, Suite 500

Northbrook, IL 60062. USA

Más información en: http://www.paho.org/English/HCP/HCT/gorgasad.htm 